\title{
Barkhausen noise from zigzag domain walls
}

\author{
B. Cerruti ${ }^{1}$ and S. Zapperi ${ }^{1}$ \\ ${ }^{1}$ CNR-INFM, Dipartimento di Fisica, Università "La Sapienza", P.le A. Moro 2, \\ 00185 Roma, Italy \\ E-mail: benedetta.cerruti@gmail.com, stefano.zapperi@roma1.infn.it
}

\begin{abstract}
We investigate the Barkhausen noise in ferromagnetic thin films with zigzag domain walls. We use a cellular automaton model that describes the motion of a zigzag domain wall in an impure ferromagnetic quasi-two dimensional sample with in-plane uniaxial magnetization at zero temperature, driven by an external magnetic field. The main ingredients of this model are the dipolar spin-spin interactions and the anisotropy energy. A power law behavior with a cutoff is found for the probability distributions of size, duration and correlation length of the Barkhausen avalanches, and the critical exponents are in agreement with the available experiments. The link between the size and the duration of the avalanches is analyzed too, and a power law behavior is found for the average size of an avalanche as a function of its duration.
\end{abstract}




\section{Introduction}

Understanding the properties of ferromagnetic thin films is still one of the open questions in the physics of magnetic systems. An important issue is linking the domains and domain walls structure of a material with its hysteretic properties, like the Barkhausen noise (BN). The Barkhausen effect 1] consists in the irregularity of the magnetization variation while magnetizing a sample with a slowly varying external magnetic field, and is due to the jerky motion of the domain walls in a system with structural disorder and impurities. Once the origin of BN was understood [2], it was soon realized that it could be used as an effective probe to investigate the magnetization dynamics in magnetic materials. Furthermore, from a purely theoretical point of view, it is a good example of dynamical critical behavior, as evidenced by experimental observation of power law distributions for the statistics of the avalanche size and duration [3]. Moreover, there is a growing evidence that soft magnetic bulk materials can be grouped in different classes according to the scaling exponents values [4, so that BN could be seen as a non destructive experimental tool for the analysis of the properties of a material, and a similar feature could be expected to hold for two-dimensional materials.

Until now, most of the models and experiments on $\mathrm{BN}$ have focused on three dimensional systems [5]. The difficulties for the study of two dimensional samples, in fact, concern both the theoretical and the experimental aspects of the problem. On the theoretical side, the topology of domain and domain walls is much more rich and complicated in two dimension (parallel or head-on domains, charged and uncharged walls, magnetization in or out the film plane, parallel or zigzag walls, labyrinthic domains, etc.) than in the bulk case [6] (mainly parallel domains with uncharged walls), so it is not obvious how to generalize the well known three dimensional models. The models currently used for three dimensional materials could be classified in two main groups, namely spin models of the Ising type [7, 8, 9, 10, and single domain wall models 11, 12, 13, 14, 15, 16. On the experimental side, the inductive experimental setup commonly used for bulk samples [5] is usually not suitable for thin films, due to the low intensity of the magnetic flux variation signal, which is roughly proportional to the sample thickness, and thus tends to vanish for very thin films. Conversely, the magnetooptical [17, 18, 19] and magneto-optical microscope magnetometry [20] experiments, though able to supply the domain structure, that is not accessible by the inductive measurements, provide only partial informations about the probed zone, and not about the whole sample. So, despite the increasing interest concerning ferromagnetic thin films applications in magnetic recording technology and spintronic devices, a complete understanding of two dimensional system behavior is still lacking.

In this article we focus on two dimensional systems with zigzag domain walls, which arise in thin films with head-on magnetization between nearest-neighbor domains, mainly due to the balance between the magnetostatic and the anisotropy contributions to the system total energy [22]. Those kind of walls have been observed for the first time in thin film magnetic recording media, where head-on domains are induced by means of 
the application of a recording head field, and have been then observed in films of several magnetic media such as iron [23, Co [24], Gd-Co 22, epitaxial Fe films grown on GaAs(001) 25, ferrite-garnet films with strong cubic anisotropy [26] and many others. This kind of walls have been observed too in ferroelectric materials, such as $\mathrm{Gd}_{2}\left(\mathrm{MoO}_{4}\right)_{3}$ crystals [27].

We present a study of the Barkhausen noise at $T=0$. We use a slightly modified version of a simple single-wall discrete model for the motion of the zigzag wall that we recently proposed for the study of the dynamic hysteresis in ferromagnetic thin films [28. Our model is based on the interplay between dipolar and anisotropy energy contributions, in the presence of structural disorder and external magnetic field. Via cellular automaton simulations the model describes the motion, in a disordered ferromagnetic thin film, of a zigzag wall between two domains of opposite magnetizations meeting head-on at the wall, up to the saturation of the magnetization driven by the external magnetic field. We find that the probability distributions of the size, the duration and the correlation length of the Barkhausen avalanches show a power law behavior with a cutoff, and the critical exponents are in quantitative agreement with experimental data two dimensional sample, as Co polycristalline thin films [20, 21]. Finally, in order to have a deeper insight on the link between the size and the duration of the avalanches, we study the behavior of the average size of an avalanches as a function of its time duration, and find that it could be described too by a power law.

\section{The model}

Our purpose is to study the motion of a single domain wall driven by an external magnetic field, by discrete model simulations. To this end, we start from a model that we have recently introduced [28]: as we are interested in the macroscopic response, the aim of our model is to discretize the zigzag wall in minimum segments in order to map the quasi-two dimensional problem of the wall motion in a one dimensional model (Fig. 1), regardless the details of the wall internal structure that are not expected to influence the macroscopic length scale.
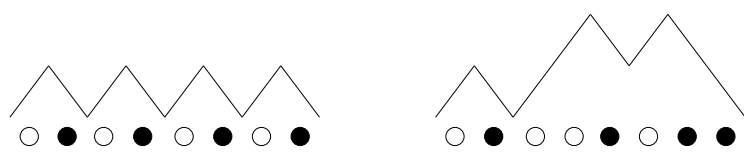

Figure 1. The mapping. Two examples of zigzag configurations. A segment with a positive (negative) slope corresponds to a void (particle).

We calculate the total energy of an arbitrary zigzag wall configuration, considering only the magnetostatic, the anisotropy and the disorder contributions, and the interaction with the external magnetic field:

$$
E=E_{m}+E_{a n}+E_{d i s}+E_{\text {ext }} .
$$


In the equation (11), the magnetostatic term $E_{m}$ takes into account the interaction between the magnetization and the stray field, the anisotropy $E_{a n}$ is the energy cost of the deviations from the easy axis of the material, that are associated with the Néel tail surrounding the wall [28, and $E_{\text {dis }}$ models structural disorder, impurities, defects etc..

As we have discussed in our previous paper [28], the magnetostatic interaction energy between two segments $i$ and $j$ could be approximated as

$$
E_{m, i j}=8 M_{s}^{2} \epsilon^{2} p^{2} \mu_{0} \frac{1}{r_{i j}}
$$

where $M_{s}$ is the saturation magnetization, $\epsilon$ is the sample thickness, $p$ is the minimal half-period of a zigzag configuration, $\mu_{0}$ is the vacuum permeability and $r_{i j}$ is the distance between the centers of mass of the two segments $i$ and $j$ (see Fig. 2).

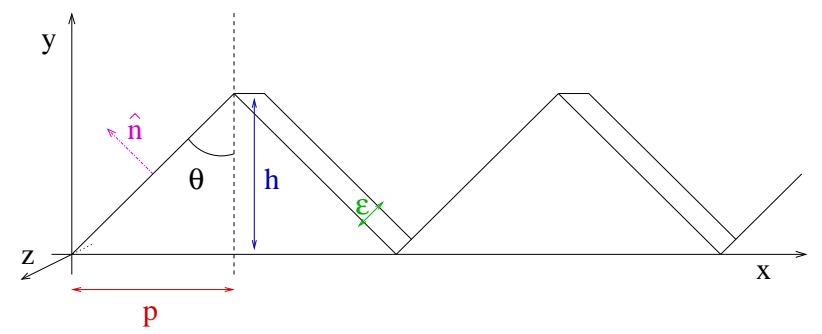

Figure 2. Sketch of the parameters of the zigzag wall. The easy axis is along the $y$ direction.

The anisotropy energy term in the simple case of an uniaxial crystal can be written as

$$
E_{a n}=\int d^{3} r K_{u} \sin ^{2} \phi
$$

where $K_{u}$ is the in-plane uniaxial anisotropy constant and $\phi$ is the angle between the easy axis and the magnetization vector. Assuming 22] that the magnetic charge associated with the magnetization rotation is uniformly distributed over the entire band containing the wall and a linear in-plane rotation of the magnetization vector, we obtain the anisotropy energy for unit length

$$
E_{a n}=\epsilon K_{u} h c(\theta),
$$

where $K_{u}$ is the anisotropy constant of the material, $h$ is the zigzag amplitude and $c(\theta)$ is a constant function of the zigzag angle $\theta$ which could be evaluated numerically (see 28. for more details).

Concerning the $E_{\text {dis }}$ term, we consider only quenched (frozen) disorder, that does not evolve on the timescale of the magnetization reversal. We model the disorder by an energy contribution associated to each site of our discretized sample which may be occupied by a segment (our discrete unit length) of the zigzag wall. This term is extracted from an uncorrelated random Gaussian distribution with zero mean. 
The interaction energy with the external magnetic field $H_{e x t}$, that is set on the easy axis direction, is given by

$$
E_{\text {ext }}=-\mu_{0} H_{\text {ext }} M,
$$

where $M$ is the magnetization of the system.

The dynamics of the model is implemented by switching on the external magnetic field $H_{\text {ext }}$, looking for all the pairs of segments with slope up-down in the wall, i.e. the void-particle pairs, and trying to exchange the positions in the pair. This rule allows only the forward motion of pairs of segments with up-down slope, and preserves the zigzag (solid-on-solid) structure of the wall. Once a possible displacement has been attempted, we calculate the total energy difference $\Delta E$ between the starting configuration and the new one by using Eq. (11). The move is accepted if $\Delta E \leq 0$, and in that case we update the configuration and continue the process, otherwise we reject it. The acceptance of the move corresponds to the starting of an avalanche, that goes on until the wall comes to rest, i.e. when the minimum difference of energy $\Delta E_{\min }$ over all the void-particle pairs is bigger than zero. To restart the process and eventually trigger another avalanche, we increase the external field by an amount $\Delta H_{e x t}=\Delta E_{\min } / \mu_{0} \Delta M$, where $\Delta M$ is the variation of the magnetization due to the flip of the void-particle pair, and continue the updating. For this parallel dynamics, we can identify the number of the iterations of the updating with the physical time. We begin the simulation from the $M=0$ at $H_{e x t}=0$ state and drive the sample to the positive saturation (our sample has a finite size).

\section{Results}

From experimental magneto-optical observations (Fig. 3) we can infer that the dynamics of the wall is jerky and proceeds by avalanches, preserving the value of the zigzag angle and with a gradual increase of the zigzag period and amplitude. These features are recovered in our simulations (see Fig. [4): the wall may be pinned by the disorder and the depinning of a void-particle pair driven by the external magnetic field could eventually trigger an avalanche. Moreover, when the field increases, the anisotropy and disorder energy terms become less important compared with $E_{\text {ext }}$. So the relevance of the magnetostatic interactions increases, and since this term tends to move the magnetostatic charges (all of the same sign at the wall) away from each other, it drives a coarsening of the zigzag segments, whose period thus increases.

An avalanche is defined as the event between two pinned configuration of the domain wall. Since in our model we only allow the forward motion of the wall, we can thus identify univocally the time duration and spatial size of an avalanche: the time duration is the number of updates from the depinning of the wall to the new pinned configuration, and the size is defined as the area interested by the magnetization reversal during an avalanche. It is thus possible to analyze the statistics of the avalanches, by constructing the probability distributions of the size $S$, the duration $T$ and the correlation length $\xi$.

As it can be seen from the figures 5 and 6 , both the distributions show a power law behavior for almost two decades, respectively $P(S) \sim S^{-\tau} f\left(S / S_{0}\right)$ for the size and 


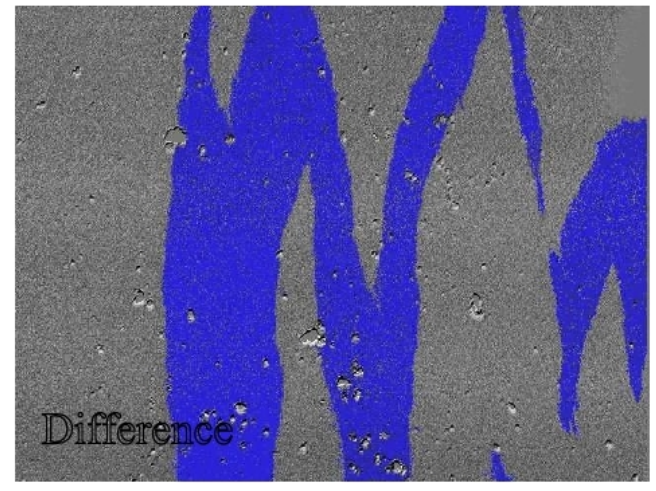

Figure 3. Magneto-optical experimental data: the colored area represents the spatial difference between the two configurations before and after an avalanche (i.e. the size of the avalanche). Courtesy of G. Durin.

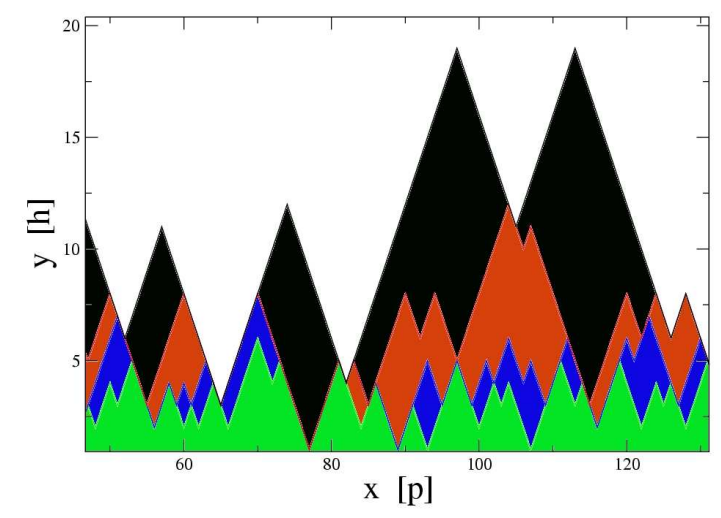

Figure 4. A zoom of four avalanches in a simulation. The colored areas represents the difference of magnetization between two successive configurations before and after an avalanche (in units of half-period and amplitude of the zigzag).

$P(T) \sim T^{-\alpha} g\left(T / T_{0}\right)$ for the time duration probability function, and a cutoff, which values are $S_{0}$ and $T_{0}$. The nature of the cutoff, which is an experimentally well known feature, is in our model due to finite size effects, as it could be seen comparing the distributions for different number of zigzag minimum segments $n$. We find that the cutoff distributions scale respectively as $S_{0} \sim L^{D}$ and $T_{0} \sim L^{\Delta}$, where $L$ is the total length of the sample, $L=n p$, and with $D \sim 2.16$ and $\Delta \sim 1.61$. In Fig. $5(b)$ and $6(b)$ we show that rescaling the variables as $S / L^{D}$ and $T / L^{\Delta}$, the rescaled probability distributions $P(S) L^{\tau D}$ and $P(T) L^{\alpha \Delta}$ obtained for the various system sizes, collapse on the same curve. The power law behavior is the fingerprint of a dynamical critical behavior, and could be characterized by the associated critical exponents. For our model, the critical exponents for the avalanches size and the duration distributions are respectively $\tau \sim 1.34$ and $\alpha \sim 1.55$, and do not essentially depend on the system size, neither on the intensity of the anisotropy and the disorder, that may beside influence the very low 


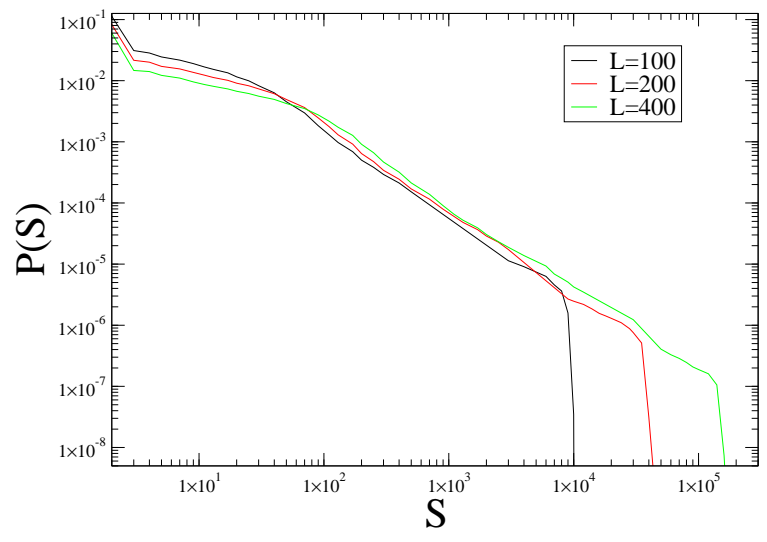

(a)

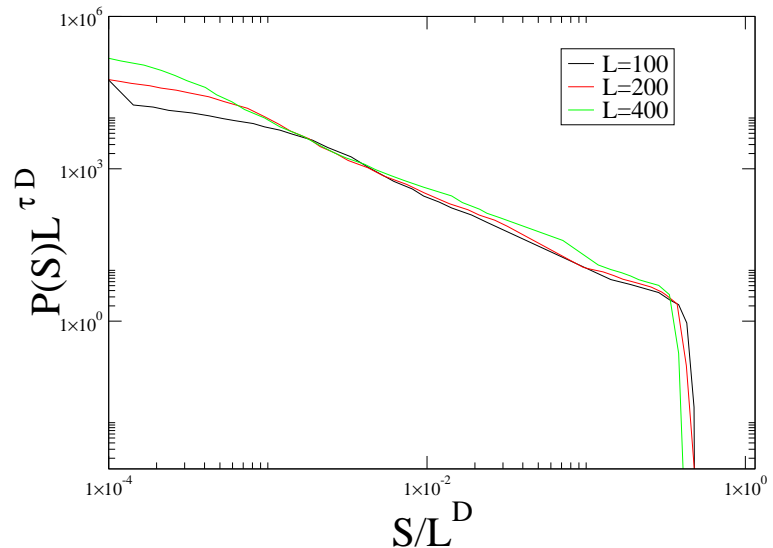

(b)

Figure 5. (a) Probability distribution for the avalanche size $S$, for three different values of the total sample length $L$. (b) The probability distribution for the rescaled variable $S / L^{D}$, where $D$ is a fitted scaling exponent which value is 2.1611 . In the power law region of the plots, they collapse on the same curve.

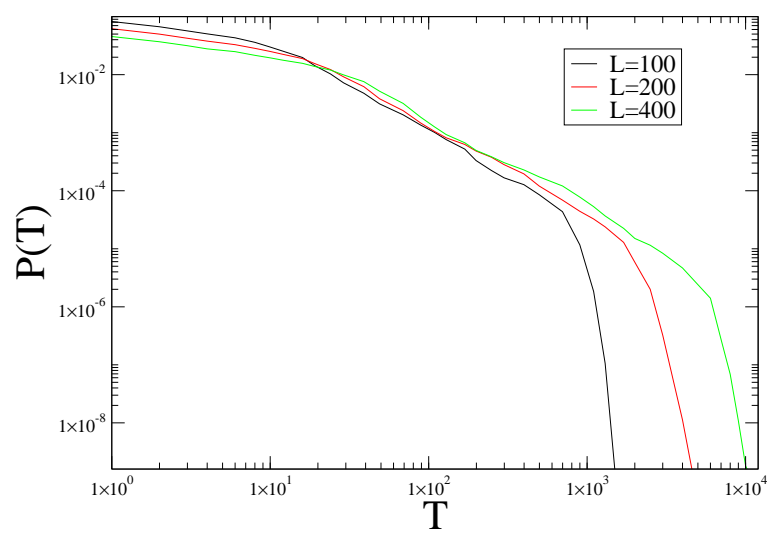

(a)

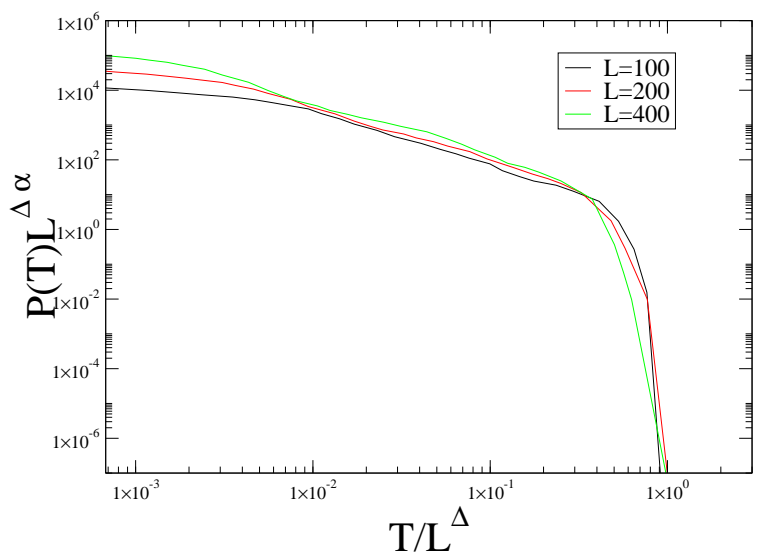

(b)

Figure 6. (a) Probability distribution for the avalanche duration $T$, for three different values of the total sample length $L$. (b) The probability distribution for the rescaled variable $T / L^{\Delta}$, where $\Delta$ is a fitted scaling exponent which value is 1.6149. In the power law region of the plots, they collapse on the same curve.

$S$ or $T$ zones of the probability distributions. The value of $\tau$ is in good agreement with recent experimental results on Co polycristalline thin films [20, 21], that give $\tau \sim 4 / 3$, although this reference does not provide informations on the duration statistics. In these references [20, 21], the authors report direct, time-resolved domain observations 
of a Barkhausen avalanche obtained by a magneto-optical microscope magnetometer, which directly visualizes the microscopic behavior of the avalanches in thin films. The observations are restricted to a limited zone of the sample for experimental reasons, and domain topology shows a system of tips, that could be interpreted as a a part of a zigzag domain wall. Anyway, more experimental confirmations would be necessary.

From our simulations we can even study the statistics of the correlation length $\xi$ of the avalanches. $\xi$ is defined as the longitudinal size of the avalanche (see Fig. 7), and gives a measure of the portion of the wall interested by the avalanche itself.

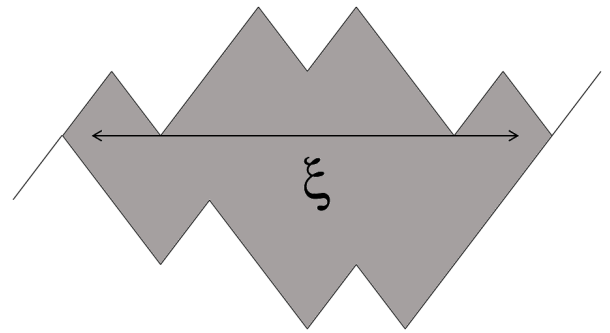

Figure 7. Definition of the correlation length in an avalanche. The gray zone represents the area interested by the avalanche.

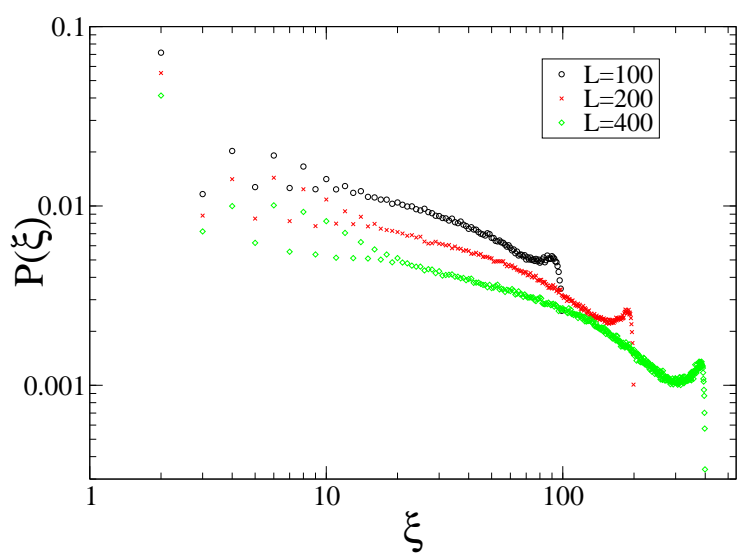

(a)

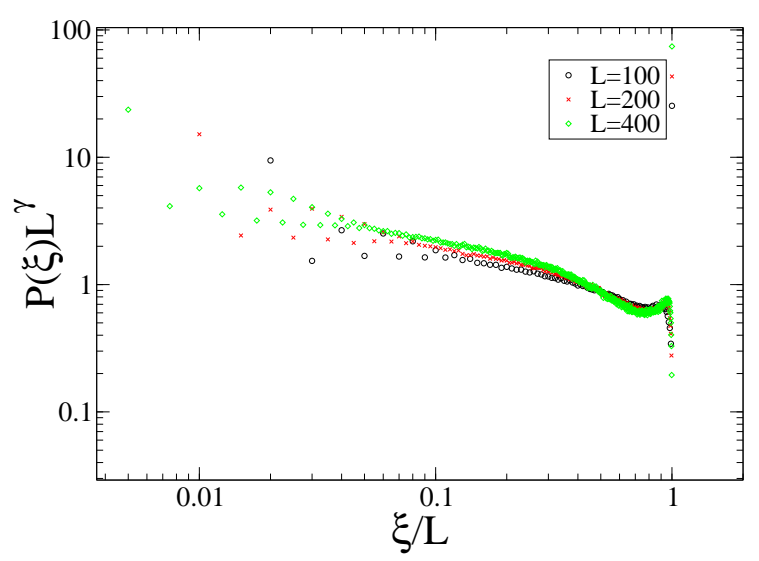

(b)

Figure 8. (a) Probability distribution for the avalanche correlation length (in unit of the minimum zigzag half period $p$, for three different values of the total sample length $L$. (b) The probability distribution for the rescaled correlation length $\xi / L$. In the power law region of the plots, they collapse on the same curve.

The probability distribution of the correlation lengths follows too a power law behavior, $P(\xi) \sim \xi^{\beta} h\left(\xi / \xi_{0}\right)$ where $\xi_{0}$ is the cutoff (Fig. 8), with an exponent close to -0.4 . The low $\xi$ zone of Fig. 8 is very noisy because of the topology of the model: small avalanches with a correlation length of an even number of minimal segment are 
more probable than avalanches with odd $\xi$, because for the correlation length study we consider the sum of the events in one uploading step, even if they are not geometrically connected. So is more likely to have, for instance, two elementary events in two separate parts of the sample than two connected events, for small avalanches size. Moreover, for very high values of $\xi$, we notice another deviation from the power law behavior, this time due to the finite size of the sample. In fact the avalanches that span the whole sample could not have a correlation length bigger than the sample size, so the probability distributions show peaks for values of $\xi$ around the system size, which are just an artifact of finite simulations, as of course the cutoff at the system length value. The cutoff distribution scales as $\xi_{0} \sim L$. In Fig. $8(b)$ we show that rescaling the correlation length as $\xi / L$, the rescaled probability distributions $P(\xi) L^{\beta}$ obtained for the various system sizes collapse on the same curve.

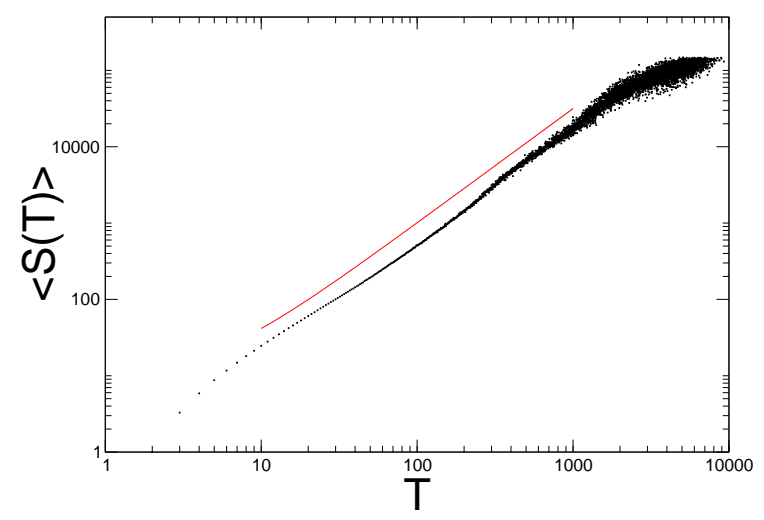

Figure 9. Average size of an avalanche vs its duration $T$ for $L=400$. A part form the cutoff at large values $T$ and effects of the discretization of the model for small $T$, the function shows a power law behavior for almost two decades with an exponent close to $\gamma \sim 1.5$ (solid line is a power law with exponent 1.45 and is a guideline to the eye).

Another interesting issue that could be studied is the correlation between the size and the duration of the avalanches. Since avalanches with the same duration could show quite different sizes, this feature could be quantified by addressing to the link between the mean size $\langle S(T)>$ of an avalanche and its duration $T$ (Fig. 9). This function follows a power law behavior $\left\langle S(T)>\sim T^{\gamma}\right.$ with an exponent close to $\gamma \sim 1.5$ for all the three sample sizes that we have investigated $(L=100, L=200$ and $L=400)$.

We can check the consistency of the exponent $\gamma$ considering that it must be $P(S) d S \sim P(T) d T$. Using the power law $P(S) \sim S^{-\tau}, P(T) \sim T^{-\alpha}$ and $\left\langle S(T)>\sim T^{\gamma}\right.$, it can be easily derived that it must be

$$
\alpha=\gamma(\tau-1)+1
$$

Using $\gamma=1.45$ and $\tau=1.34$ we would obtain $\alpha=1.49$ that is in reasonable agreement with the measured value $\alpha=1.55$. 


\section{Conclusions}

The Barkhausen noise is known to be due to the jerky motion of the domain walls in a disordered material during the magnetization process. Even if the essential physics of the problem is well understood, many questions are still open. One of the most challenging related topics is the physics of ferromagnetic thin films, which displays new features with respect to bulk materials, and still remains to be fully explored both experimentally and theoretically. In this work we have applied a slightly modified version of a model that we have recently proposed [28] for the study of dynamic hysteresis for systems with zigzag domain walls. This model takes into account the contribution to the total energy of the dipolar long range interactions, the anisotropy and the disorder. The dynamics of the model describes qualitatively the experimentally observed features of the domain wall motion, like the jerky nature of the motion and the coarsening of the zigzag amplitude. We have studied the size, the duration and the correlation length of the avalanches by means of their probability distributions, via cellular automaton simulations. All these distributions show a power law behavior and a cutoff due to finite size effects. The critical exponents of the three distributions are derived and we find a good agreement for the value of $\tau \sim 1.34$, associated with the size statistics, with recent magneto-optical measurements on Co polycristalline thin films [20]. Anyway, more experimental confirmations are needed, especially for the duration and the correlation length distributions, for which no experimental data are available up to now. Finally, we have investigated the link between the size and the duration of the Barkhausen avalanches, by studying the average size of an avalanche as a function of its duration $T$. Even this function follows a power law, with a critical exponent close to 1.5.

[1] Barkhausen H, 1919 Z. Phys. 20401

[2] Williams H J and Shockley W, 1949 Phys. Rev. 75178

[3] Sethna J P, Dahmen K A and Myers C R, 2001 Nature 410087203

[4] Durin G and Zapperi S, 2000 Phys. Rev. Lett. 844705

[5] Durin G and Zapperi S, 2005 The Science of Hysteresis: Physical Modeling, Micromagnetics, and Magnetization Dynamics vol II (Amsterdam: Academic) chapter III (The Barkhausen Noise) pp 181-267 cond-mat/0404512

[6] Hubert A and Schäfer R, 1998 Magnetic domains (Springer: New York )

[7] Sethna J P, Dahmen K A, Kartha S, Krumhansl J A, Roberts B W and Shore J D, 1993 Phys. Rev. Lett. 703347

[8] Vives E and Planes A, 1994 Phys. Rev. B 503839

[9] Vives E and Planes A, 2000 J. Mag. Magn. Mat. 221164

[10] Vives E and Planes A, 2001 Phys. Rev. B 63134431

[11] Cizeau P, Zapperi S, Durin G and Stanley H E, 1997 Phys. Rev. Lett. 794669

[12] Zapperi S, Cizeau P, Durin G and Stanley H E, 1998 Phys. Rev. B 586353

[13] Urbach J S, Madison R C, Markert J T, 1995 Phys. Rev. Lett. 75276

[14] Narayan O, 1996 Phys. Rev. Lett. 773855

[15] Bahiana M, Koiller B, de Queiroz S L A, Denardin J C and Sommer R L, 1999 Phys. Rev. E 59 
3884

[16] de Queiroz S L A and Bahiana M, 2001 Phys. Rev. E 64066127

[17] Walsh B, Austvold S and Proksch R, 1998 J. Appl. Phys. 845709

[18] Puppin E, 2000 Phys. Rev. Lett. 845415

[19] Schwarz A, Liebmann M, Kaiser U, Wiesendanger R, Noh T W and Kim D W, 2004 Phys. Rev. Lett. 92077296

[20] Kim D -H, Choe S -B and Shin S -C, 2003 Phys. Rev. Lett. 90330

[21] Kim D -H, Choe S -B and Shin S -C, 2003 J. Appl. Phys 936564

[22] Freiser M J, 1979 IBM J. Res. Dev. 23330

[23] Curland N and Speliotis D E, 1970 J. Appl. Phys. 411099

[24] Dressler D and Judy J, 1974 IEEE Trans. Magn. MAG 10674

[25] Lee W Y, Choi B -Ch, Xu Y B and Bland J A C, 1999 Phys. Rev. B 6010216

[26] Vlasko -Vlasov V K, Dedukh L M and Nikitenko V I, 1976 Sov. Phys. JETP 441208

[27] A.N. Alexeyev, 1996 Appl. Phys. Lett. 68 (2) 159

[28] Cerruti B and Zapperi S, 2006 Dynamic hysteresis from zigzag domain walls Preprint cond-mat/0603406 